\title{
Cinética de Passagem da Digesta, Balanço Hídrico e de Nitrogênio em Eqüinos Consumindo Dietas com Diferentes Proporções de Volumoso e Concentrado1
}

\author{
Chiara Albano de Araújo Oliveira ${ }^{2}$, Fernando Queiroz de Almeida ${ }^{3}$, Antônio Assis Vieira ${ }^{3}$, Ângela \\ Maria Quintão Lana ${ }^{3}$, Robert Macedo ${ }^{4}$, Bruna Adese Lopes ${ }^{5}$, Anderson Corassa ${ }^{6}$
}

\begin{abstract}
RESUMO - Este trabalho foi conduzido com os objetivos de avaliar a cinética de passagem da digesta no trato digestivo e estimar o balanço hídrico e de nitrogênio em eqüinos consumindo dietas com diferentes níveis de volumoso. Foram utilizados quatro potros não castrados, mestiços, com idade de 18 meses, alojados em baias individuais e exercitados uma vez ao dia. O delineamento experimental utilizado foi o quadrado latino 4 x 4, em que cada período experimental teve duração de 14 dias de adaptação à dieta e cinco dias de coleta fecal. As dietas foram compostas por feno de capim-coastcross e concentrado, fornecidos simultaneamente, duas vezes ao dia, nas proporções de 40:60, 60:40, 80:20 e 100:0. A cinética de passagem da digesta foi estimada pelo tempo médio de retenção da digesta (TMR), da taxa de passagem (TxP) e do tempo de trânsito (TT), utilizando dois indicadores externos, o cromo fixado como mordente na fibra em detergente neutro (Cr-FDN) e o complexo cobalto-EDTA (Co-EDTA). O balanço hídrico foi avaliado de acordo com as quantidades de água ingerida in natura e na dieta e excretada na urina e nas fezes. O balanço de nitrogênio foi avaliado de acordo com as quantidades diárias de compostos nitrogenados consumidos e excretados. As médias dos valores da cinética de passagem nas fases sólida e líquida da digesta foram 42,7 e 33,8 horas para TMR e de 2,4 e 3,1 \%/hora para TxP. O TT da fase líquida da digesta apresentou efeito linear negativo, em função da inclusão de volumoso na dieta. O consumo médio de água foi de 13,8 litros. O balanço hídrico variou de 7,9 a 1,8 litros para as dietas com 40 e $100 \%$ de volumoso, respectivamente, reduzindo de forma linear, com a inclusão do volumoso na dieta.
\end{abstract}

Palavras-chave: balanço hídrico, eqüinos, nitrogênio, taxa de passagem

\section{Kinetics of Passage of Digesta and Water and Nitrogen Balance in Horses Fed Diets with Different Ratios of Roughage and Concentrate}

\begin{abstract}
This work aims to evaluate the passage of digesta kinetics through the digestive tract and estimate the water and nitrogen balance in horses fed different levels of roughage in diets. Four crossbred yearling were used with age of 18 months, housed in individual stalls and exercised once a day. The present experiment was performed according to a 4 X 4 Latin Square design which each experimental period had 14 days for adaptation to diet and five days for fecal collection. Diets were composed by coastcross bermudagrass hay and concentrate, fed simultaneously and supplied twice a day, in proportions of 40:60, 60:40, 80:20 and 100:0. Kinetics of digesta passage was evaluated through the medium retention time (MRT), rate of passage (RP) and transit time (TT), using two external markers, mordant chromium in neutral detergent fiber (Cr-NDF) and Cobalt-EDTA (Co-EDTA). Water balance was evaluate according to water intake in natura and in diets and water excreted in urine and feces. Nitrogen balance was evaluated with daily intake and excreted nitrogen compounds. The MRT and RP of solid and liquid phases of digesta were 42.7 and 33.8 hours and 2.4 and 3.1\%/hours, respectively. Transit time had a negative linear effect in function of roughage inclusion in diets. Daily average water intake was 13.8 liters and water balance was 7.9 liters in diet with $40 \%$ of roughage and 1.8 liters in diet with $100 \%$ of roughage, showing a linear reduction with roughage inclusion in diets.
\end{abstract}

Key Words: horse, nitrogen, rate of passage, water balance

\section{Introdução}

Os cavalos são capazes de utilizar grandes quantidades de forragem para atender as suas exigências nutricionais. Atualmente, para maximizar o crescimento e a produtividade dos eqüinos, as dietas con- têm altos níveis de grãos e suplementos, portanto, este tipo de manejo nutricional ainda oferece uma significante contribuição da dieta volumosa no atendimento dos requerimentos nutricionais dos animais.

De acordo com Olsson \& Ruudvere (1955) e Meyer (1995), vários fatores afetam o tempo de

\footnotetext{
${ }^{1}$ Parte da Dissertação do primeiro autor apresentada ao Programa de Pós-graduação em Zootecnia da UFRRJ. Projeto de pesquisa financiado pela FAPERJ.

2 Discente de Mestrado em Zootecnia-UFRRJ. E.mail: chiara@zipmail.com

3 Professor Adjunto. UFRRJ. E.mail: falmeida@ufrrj.br

Correspondência: DMCV-IV-UFRRJ. BR 465, km 7, Seropédica, RJ. CEP: 23851-970

4 Discente de Doutorado em Ciências do Solo-UFRRJ.

5 Bolsista de Iniciação Científica - PIBIC/CNPq-UFRRJ.

6 Bolsista de Iniciação Científica - FAPERJ
} 
permanência dos alimentos nos diversos segmentos do trato digestivo dos eqüinos, alterando, conseqüentemente, sua digestibilidade: a individualidade do animal, a composição química e quantidade do alimento, o tipo de atividade física do animal, o tipo e tamanho das partículas do alimento e a quantidade de fibra presente na dieta.

Segundo Udén et al. (1982), o tempo de retenção da digesta no trato gastrointestinal é significativamente menor em cavalos, quando comparado com ruminantes. Wanderley et al. (1992) observaram que a taxa de passagem da digesta está estrategicamente relacionada com a alimentação dos herbívoros e pode ser avaliada com o uso de indicadores.

A ingestão de água é avaliada em relação à matéria seca e energia ingerida, enquanto sua excreção está associada ao conteúdo de proteínas, minerais e fibra da dieta. Existe perda contínua de fluidos do corpo pelas fezes, pela urina, sudorese e evaporação pela respiração. Estas perdas têm que ser compensadas pela ingestão de água e, ou, água dos ingredientes da dieta. Fatores conhecidos que afetam a ingestão de água pelos eqüinos são a composição da dieta, temperatura da água, palatabilidade da água e exercícios (Cymbaluk, 1989).

A idade e a utilização do cavalo são as considerações mais importantes na determinação das necessidades de proteína. Existem ainda outros fatores importantes que devem ser avaliados na seleção de uma dieta para uma fase em particular da vida de um eqüino, destacando-se a digestibilidade da proteína, o conteúdo de aminoácidos da proteína e a relação entre proteína e energia na dieta, especialmente na alimentação de cavalos em crescimento. Existem vários tipos diferentes de suplementação protéica para eqüinos e, em geral, a fonte de proteína utilizada na suplementação de eqüinos é de alta qualidade, como, por exemplo, o farelo de soja, a alfafa e a proteína do leite (Pagan, 2001).

De acordo com Glade \& Bell (1981), existe uma relação entre a habilidade dos eqüinos em utilizar a uréia da dieta, a fermentação cecal e o metabolismo do nitrogênio microbiano. Vander Noot \& Gilbreath(1970) sugeriram que existe uma relação inversa entre o conteúdo de fibra bruta e a digestibilidade aparente do nitrogênio nos eqüinos e, segundo Glade (1984), alimentando eqüinos com uma dieta composta por milho, soja, feno e uréia, houve redução na eficiência da utilização do nitrogênio, com o aumento da excreção de água nas fezes e do nitrogênio metabólico e endógeno.
Este estudo foi conduzido com os objetivos de avaliar a cinética de passagem da digesta pelo trato digestivo e estimar o balanço hídrico e de nitrogênio, em eqüinos consumindo dietas com diferentes níveis de volumoso.

\section{Materiais e Métodos}

O experimento foi conduzido na Universidade Federal Rural do Rio de Janeiro, Seropédica, RJ, no período de setembro a novembro de 2000 , com uma temperatura média de $22,5^{\circ} \mathrm{C}$. O ensaio de digestão foi realizado em quatro baias individuais de $3 \mathrm{~m}^{2}$, pé direito de $2 \mathrm{~m}$, de alvenaria, com piso de cimento, janelas, providas de comedouro para ração e bebedouro.

Foram utilizados quatro potros mestiços, com peso vivo médio de $200 \mathrm{~kg}$, não castrados, média de idade de 18 meses, submetidos ao mesmo manejo: imunizados contra tétano, vermifugados, pulverizados contra ectoparasitos e submetidos à doma de baixo. O período experimental teve duração de 19 dias, com 14 dias de adaptação às dietas e cinco dias de coleta de fezes. Durante este período, os animais foram exercitados, ao passo e trote, uma vez ao dia, por 30 minutos, no período da manhã.

As dietas foram formuladas considerando-se o consumo diário de $2,0 \%$ do peso vivo dos animais, com base na matéria seca, de acordo com as recomendações do NRC (1989), compostas por feno de capim-coastcross (Cynodon dactlon cv. Coastcross) como volumoso e concentrado de modo a fornecer as proporções de volumoso e concentrado de 40:60, 60:40, 80:20 e 100:00, que compuseram os tratamentos $1,2,3$ e 4 . O feno de coastcross foi fornecido picado, duas vezes ao dia, às $7 \mathrm{e} 19 \mathrm{~h}$, simultaneamente com o concentrado, em quantidades iguais, exceto no tratamento 4.

A composição das dietas, os teores de energia bruta (EB) e os percentuais de proteína bruta (PB), fibra em detergente neutro (FDN) e fibra em detergente ácido (FDA) estão apresentados na Tabela 1.

A avaliação da cinética de passagem da digesta em todo o trato digestivo foi feita por intermédio da estimativa do tempo médio de retenção da digesta (TMR), da taxa de passagem (TxP) e do tempo de trânsito (TT). Foram utilizados dois indicadores externos, o cromo fixado como mordente na fibra em detergente neutro (Cr-FDN), utilizado na estimativa da cinética de passagem da fase sólida da digesta, e o complexo cobaltoEDTA (Co-EDTA), usado na estimativa da cinética de

R. Bras. Zootec., v.32, n.1, p.140-149, 2003 
Tabela 1 - Composição percentual dos ingredientes, teores de energia bruta (EB) e percentuais de proteína bruta (PB), fibra em detergente neutro (FDN) e fibra em detergente ácido (FDA), expressos na matéria seca, nas dietas experimentais

Table 1 - Percentage composition of ingredients, gross energy (GE), crude protein (CP), neutral detergent fiber (NDF) and acid detergent fiber (ADF), on dry matter basis, of the experimental diets

\begin{tabular}{|c|c|c|c|c|}
\hline \multirow[t]{2}{*}{$\begin{array}{l}\text { Ingredientes/Nutrientes } \\
\text { Ingredients/Nutrients }\end{array}$} & \multicolumn{4}{|c|}{$\begin{array}{l}\text { Proporção volumoso : concentrado } \\
\text { Proportion roughage : concentrate }\end{array}$} \\
\hline & 40:60 & $60: 40$ & $80: 20$ & 100:00 \\
\hline Feno de capim-coastcross & 40 & 60 & 80 & 100 \\
\hline Coastcross hay & & & & \\
\hline Milho - grão & 43,5 & 26,4 & 7,0 & 0 \\
\hline Corn & & & & \\
\hline Farelo de soja & 14,5 & 12,0 & 11,2 & 0 \\
\hline Soybean meal & & & & \\
\hline $\begin{array}{l}\text { Suplemento mineral-vitamínico* } \\
\text { Mineral-vitamin mixture }\end{array}$ & 1,0 & 0,8 & 0,8 & 0 \\
\hline Sal & 1,0 & 0,8 & 1,0 & 0 \\
\hline Salt & & & & \\
\hline Total & 100 & 100 & 100 & 100 \\
\hline MS (\%) & 90,1 & 89,6 & 90,4 & 91,1 \\
\hline$D M$ & & & & \\
\hline $\mathrm{EB}(\mathrm{Mcal} / \mathrm{kg})$ & 4,6 & 4,6 & 4,4 & 4,6 \\
\hline$G E$ & & & & \\
\hline $\mathrm{PB}(\%)$ & 12,5 & 13,3 & 12,1 & 7,3 \\
\hline$C P$ & & & & \\
\hline FDN (\%) & 37,1 & 50,0 & 63,0 & 75,7 \\
\hline$N D F$ & & & & \\
\hline FDA $(\%)$ & 19,1 & 26,6 & 33,7 & 40,6 \\
\hline$A D F$ & & & & \\
\hline
\end{tabular}

passagem da fase líquida da digesta.

O Cr-FDN e o Co-EDTA foram preparados segundo método descrito por Udén et al. (1980), modificado por Gomes (1991). Os indicadores foram fornecidos aos animais no $15^{\circ}$ dia de cada período experimental. O Cr-FDN foi fornecido em dose única de $30 \mathrm{~g}$ e o Co-EDTA, em dose única de $3 \mathrm{~g}$, juntamente com a dieta fornecida às $7 \mathrm{~h}$. Amostras de fezes, de aproximadamente $200 \mathrm{~g}$, foram coletadas diretamente do reto dos animais às $0,4,8,12,16,20$, $24,30,36,42,48,54,60,66,72$ e 78 horas após o fornecimento dos indicadores, segundo metodologia descrita por Cuddeford et al. (1992).

Os teores de $\mathrm{Cr}$ e Co foram determinados nas amostras de fezes, segundo metodologia descrita por Kimura \& Miller (1957) e Bolin \& King (1952).

O tempo médio de retenção da digesta em todo o trato digestivo foi calculado pela equação proposta por Faichney (1975):

$$
\operatorname{TMR}(\text { horas })=\frac{\sum \mathrm{mix} \mathrm{ti}}{\sum \mathrm{mi}}
$$

em que mi é a quantidade de indicador excretado no $\mathrm{i}$ intervalo de tempo e ti, o intervalo de tempo entre o fornecimento do indicador e i tempo de coleta fecal.

A taxa de passagem da digesta em todo o tubo digestivo foi estimada como o inverso da TMR:

$$
\operatorname{TxP}\left(\% h^{-1}\right)=1 / \mathrm{TMR}
$$

O tempo de trânsito da digesta foi considerado como o intervalo de tempo entre o fornecimento do indicador aos animais e o aparecimento inicial nas fezes.

A água foi fornecida em recipiente de volume conhecido, renovada quatro vezes ao dia, e as sobras, medidas diariamente, para o cálculo do consumo médio diário. No $19^{\circ}$ dia de cada período experimental, a urina foi coletada durante 24 horas, com o auxílio de coletores de urina para eqüinos, conforme descrito por Tasker(1966), em baldes de plástico contendo $25 \mathrm{~mL}$ de ácido clorídrico. A quantidade total de urina excretada por cada animal foi medida, sendo retiradas amostras de aproximadamente $300 \mathrm{~mL}$, armazenadas a $-10^{\circ} \mathrm{C}$, para posterior análise de nitrogênio.

O balanço hídrico foi avaliado utilizando as seguintes equações, segundo Cymbaluk (1989): 
Consumo Total de Água (litros/dia) = Água consumida + Água consumida na dieta

Excreção Total de Água (litros/dia) = Água urina (\% MS) + Água fezes (\% MS)

Balanço Hídrico (litros/dia) = Consumo total água Excreção total água

O balanço de nitrogênio foi avaliado de acordo com as quantidades diárias de compostos nitrogenados consumidos e excretados nas fezes e urina. Os teores de nitrogênio $(\mathrm{N})$ nas dietas, fezes e urina foram determinados pelo método de Kjeldahl, segundo Silva (1990). As quantidades de compostos nitrogenados retidos e absorvidos foram calculadas utilizando as seguintes equações, segundo Almeida (1997):

$\mathrm{N}$ retido $(\mathrm{g} / \mathrm{dia})=\mathrm{N}$ consumido $(\mathrm{g})-\mathrm{N}$ fecal $(\mathrm{g})-\mathrm{N}$ urinário $(\mathrm{g})$

$\mathrm{N}$ absorvido $(\mathrm{g} / \mathrm{dia})=\mathrm{N}$ consumido $(\mathrm{g})-\mathrm{N}$ fecal $(\mathrm{g})$

Foi utilizado o delineamento experimental em quadrado latino $4 \times 4$, sendo quatro tratamentos, quatro animais; $o$ animal na repetição foi considerado como unidade experimental. Os valores obtidos foram submetidos às análises de variância e regressão, utilizando-se o programa SAEG - Sistema para Análises Estatísticas e Genéticas (UFV, 1997), e os modelos foram escolhidos com base no teste dos coeficientes de regressão, adotando-se o nível de 5\% de probabilidade, pelo teste $t$, e do coeficiente de determinação. Os valores médios de TMR, TxP e TT das fases sólida e líquida da digesta foram comparados entre si em cada variável pelo teste de $\mathrm{F}$, adotando-se o nível de $5 \%$ de probabilidade.

\section{Resultados e Discussão}

Os valores médios da cinética de passagem da digesta, nas fases sólida e líquida, avaliadas pelo tempo médio de retenção, pela taxa de passagem e pelo tempo de trânsito ao longo do trato digestivo, em função do nível de volumoso nas dietas, podem ser observados na Tabela 2.

Não houve efeito significativo do nível dietético de volumoso no tempo médio de retenção, na taxa de passagem nas fases sólida de líquida da digesta e no tempo de trânsito da fase sólida da digesta, porém houve redução linear $(\mathrm{P}<0,05)$ no tempo de trânsito da fase líquida da digesta, em função do aumento do nível de volumoso na dieta, indicando que o tempo de trânsito da fase líquida da digesta é reduzido com o aumento no nível de fibra na dieta.

Fombelle et al. (1999), avaliando a cinética de passagem da digesta em eqüinos consumindo dietas exclusivas de volumosos e mistas, observaram diferenças significativas do TMR nos animais consumindo dietas exclusivas em feno, com a adição de $50 \%$ de grãos, sendo a passagem da digesta pelo trato digestivo mais rápida na dieta exclusiva de volumoso, de 30,1 horas, do que na dieta mista, de 42,2 horas. Não se observaram diferenças significativas entre o TMR das fases sólida e líquida, com valores de 34,9 e 30,1 horas, para a dieta exclusiva em volumoso, e de 42,9 e 42,2 horas, para a dieta mista, respectivamente.

Em relação ao processamento do volumoso da dieta, Drogoul et al. (2000) utilizaram feno moído e peletizado na alimentação de pôneis e observaram, entre as duas formas de apresentação do feno, aumento significativo no tempo médio de retenção da digesta na fase sólida, que foi de 42,2 horas para o feno moído e de 50,4 horas para o feno peletizado. Este fenômeno pode ser resultado de menor atividade celulolítica sobre a digestão da dieta peletizada.

A taxa de passagem da digesta da fase sólida medida com o cromo mordante de cada dieta - e da fase líquida da digesta - medida com o cobalto EDTA de cada dieta -, em função do nível de volumoso, está apresentada nas Figuras 1 e 2.

Pagan et al. (1998) observaram o tempo médio de

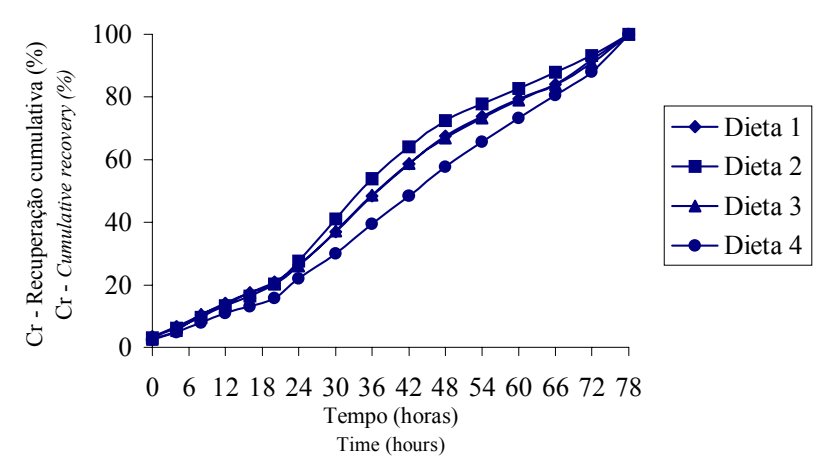

Figura 1 - Taxa média de recuperação cumulativa do cromo em dietas para eqüinos com diferentes níveis de volumoso.

Figure 1 - Mean cumulative of chromium recovery rates in horses fed diets with different levels of roughage.

R. Bras. Zootec., v.32, n.1, p.140-149, 2003 
Tabela 2 - Média e equações de regressão (ER) dos valores do tempo médio de retenção (TMR), da taxa de passagem (TxP) e do tempo de trânsito (TT), nas fases sólida e líquida da digesta, em dietas com diferentes níveis de volumoso

Table 2 - Averages and regression equations (RE) of mean retention time (MRT), rate of passage (RP) and transit time (TT) of solid and liquid phase of digesta, in diets with different levels of roughage

\begin{tabular}{|c|c|c|c|c|c|c|}
\hline \multirow[t]{2}{*}{$\begin{array}{l}\text { Variável } \\
\text { Variable }\end{array}$} & & \multicolumn{4}{|c|}{$\begin{array}{l}\text { Proporção volumoso : concentrado } \\
\text { Proportion roughage : concentrate }\end{array}$} & \multirow{2}{*}{$\begin{array}{c}\mathrm{ER} \\
\text { Regression } \\
\text { equation }\end{array}$} \\
\hline & & $40: 60$ & $60: 40$ & $80: 20$ & $100: 00$ & \\
\hline TMR (horas) & $\begin{array}{l}\text { Fase sólida } \\
\text { Solid phase }\end{array}$ & 45,7 & 42,3 & 40,2 & 42,3 & $\hat{\mathrm{Y}}=42,7 \mathrm{a}$ \\
\hline MRT (hours) & $\begin{array}{l}\text { Fase líquida } \\
\text { Liquid phase }\end{array}$ & 37,7 & 33,2 & 31,5 & 32,8 & $\hat{\mathrm{Y}}=33,8 \mathrm{~b}$ \\
\hline $\operatorname{TxP}(\% /$ hora $)$ & $\begin{array}{l}\text { Fase sólida } \\
\text { Solid phase }\end{array}$ & 2,2 & 2,4 & 2,5 & 2,4 & $\hat{\mathrm{Y}}=2,4 \mathrm{~b}$ \\
\hline$R P(\%$ hour $)$ & $\begin{array}{l}\text { Fase líquida } \\
\text { Liquid phase }\end{array}$ & 2,8 & 3,1 & 3,2 & 3,1 & $\hat{\mathrm{Y}}=3,0 \mathrm{a}$ \\
\hline TT (horas) & $\begin{array}{l}\text { Fase sólida } \\
\text { Solid phase }\end{array}$ & $27,0 \mathrm{a}$ & $25,5 \mathrm{a}$ & $23,0 \mathrm{a}$ & $27,0 \mathrm{a}$ & $\hat{\mathrm{Y}}=25,6$ \\
\hline$T T$ (hours) & $\begin{array}{l}\text { Fase líquida } \\
\text { Liquid phase }\end{array}$ & $14,0 \mathrm{~b}$ & $12,0 \mathrm{~b}$ & $6,0 \mathrm{~b}$ & $4,0 \mathrm{~b}$ & 1 \\
\hline
\end{tabular}

Médias seguidas de letras distintas na coluna, para cada variável, diferem entre si pelo teste $F(P<0,05)$.

Means followed by different letters within a column are different by $F$ test $(P<.05)$.

$1-\hat{Y}=21,6-0,18 X ; r^{2}=95,0$

$\mathrm{X}=$ Nível de volumoso.

$X=$ Level of roughage.

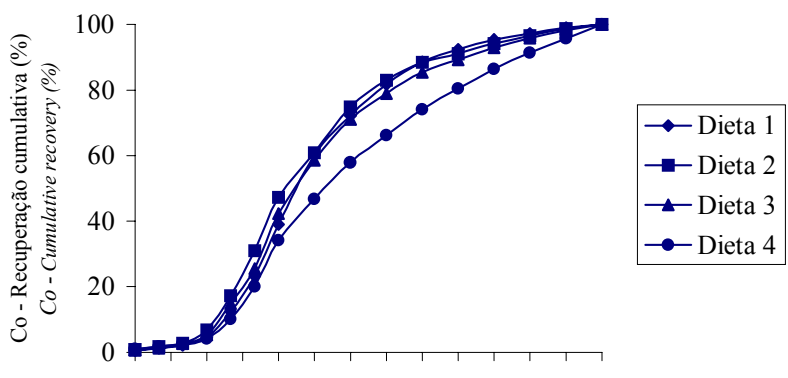

$\begin{array}{lllll}0 & 6 & 12 & 1824303642485460667278\end{array}$ Tempo (horas)

Time (hours)

Figura 2 - Taxa média de recuperação cumulativa do cobalto em dietas com diferentes níveis de volumoso, para eqüinos.

Figure 2 - Mean cumulative of cobalt recovery rates in horses fed diets with different levels of roughage.

retenção da digesta de 32,3 e 41,6 horas, em eqüinos alimentados com dietas contendo apenas volumoso ou mistas com volumoso e concentrado, respectivamente. Yonder et al. (1997) observaram que eqüinos alimentados com dietas contendo alto nível de volumoso tiveram tempo médio de retenção de 42,8 horas, menor que aqueles alimentados com dietas contendo baixo nível de fibra. Estes resultados estão de acordo com os obtidos no presente trabalho, em que o tempo médio de retenção foi de 42,3 horas, para a dieta exclusiva em volumoso.

Pearson \& Merritt (1991), alimentando pôneis com dietas exclusivas de feno ou pallha e avaliando a cinética de passagem da digesta por intermédio dos indicadores das fases sólida (Cr-FDN) e líquida (CoEDTA) da digesta, observaram valores de 29,9 e 34,8 horas para TMR da fase fase sólida e de 31,3 e 34,1 horas para TMR da fase líquida nos animais consumindo feno e palha, respectivamente. No presente experimento, os valores observados de TMR na dieta exclusiva de volumoso foram de 42,7 horas na fase sólida, superior aos observados pelos autores citados anteriormente, e de 33,8 horas na fase líquida, que são semelhantes aos observados pelos autores citados.

Cuddeford et al. (1995), utilizando dietas mistas de alfafa e palha de aveia para equinos, observaram valores para TMR de 44,2 e 45,4 horas para as fases sólida e líquida da digesta, estimadas com os indicadores Cr-FDN e Co-EDTA, respectivamente. Valores superiores aos observados no presente experimento de 42,7 e 32,8 horas para TMR nas fases sólida e líquida, respectivamente, enquanto Tood et al. (1995), utilizando alfafa em cubos na alimentação de eqüinos, 
estimaram a cinética de passagem utilizando-se disprósio, fornecido diariamente, e observaram tempo médio de retenção da fase líquida da digesta de 24,6 horas. No presente trabalho, o tempo médio de retenção observado na dieta exclusiva de volumoso foi de 32,8 horas para fase líquida, superior àqueles observados pelos autores citados anteriormente.

Geralmente, o aumento no tempo médio de retenção das partículas contribui para melhor digestão da fibra. No presente experimento, observou-se que, com a elevação do percentual de volumoso nas dietas, não houve aumento significativo no TMR da digesta, sem alteração na degradação da fibra, o que pode ser explicado pelos valores médios dos coeficientes de digestibilidade da FDN de $46,7 \%$ e da FDA de $36,9 \%$, que não apresentarem diferenças significativas em função do nível de volumoso nas dietas.

Wanderley et al. (1992), utilizando o Cr-FDN e o Co-EDTA em dose única como indicadores de passagem da digesta em eqüinos, estimaram a TxP em $10,8 \%$ por hora para a fase sólida da digesta e de $10,6 \%$ por hora para a fase líquida da digesta, valores muito superiores aos observados no presente experimento, de $2,4 \%$ por hora para a fase sólida e de 3,0\% por hora para a fase líquida.

Segundo Hintz et al. (1973), os alimentos peletizados têm taxa de passagem mais rápida que os alimentos volumosos fenados, moídos ou inteiros, enquanto os alimentos volumosos verdes têm taxa de passagem menor pelo trato gastrointestinal que os alimentos volumosos fenados (Olsson \& Ruudvere, 1955). Holland et al. (1998), alimentando eqüinos com duas dietas, uma exclusiva de feno de alfafa e a outra composta de feno de alfafa e concentrado peletizado, verificaram tempo de trânsito de 32,7 horas para fase sólida da digesta, em dieta exclusiva de feno, e de 18,1 horas para a dieta mista. No presente trabalho, o tempo de trânsito da digesta para a dieta exclusiva em volumoso foi de 27,0 horas, menor que o observado pelos autores citados, e os tempos de trânsito observados para as dietas mistas com proporções de 20,60 e $80 \%$ de volumoso foram de 27,0; 25,5; e 23,0 horas, respectivamente, valores maiores que os observados pelos citados autores.

Segundo Tood et al. (1995), alimentando eqüinos com alfafa em cubos, o tempo de trânsito observado para a fase líquida da digesta foi de 7,6 horas, valor próximo àqueles observados para a fase líquida da digesta no presente trabalho, que foram de 14, 12, 6 e 4 horas, para dietas com proporção de volumoso de
40, 60, 80 e $100 \%$ nas dietas, respectivamente.

Os valores médios diários do consumo total e da excreção urinária e fecal de água e o balanço hídrico são apresentados na Tabela 3.

Não houve diferença significativa no consumo diário de água, expresso em litros ou em litros por $\mathrm{kg}$ de MS ingerida, com médias de 13,82 litros/dia e 3,88 litros/kg de MS ingerida. Assim, o nível de volumoso na dieta não influenciou a ingestão de água, discordando dos resultados obtidos por Meyer (1987), que, avaliando cavalos alimentados com dietas exclusivas de feno, observou maior ingestão de água, em comparação aos animais alimentados com dietas mistas.

A ingestão de água está relacionada com o consumo de matéria seca ou da quantidade total de alimento ingerido, podendo variar com o tipo e o processamento do volumoso e da ração concentrada ingerida, porque o conteúdo de minerais, fibra e outros componentes é diferente entre os alimentos, afetando o consumo de água (Cunha, 1991). Segundo o NRC (1989), animais consumindo dietas exclusivas de feno ingerem água em uma proporção de 3,6 litro de água para cada $\mathrm{kg}$ de MS consumida, enquanto, em animais alimentados com dietas compostas por feno e concentrado, esta proporção é de $2,9: 1$, recomendando-se o consumo de 2 a 3 litros de água $/ \mathrm{kg}$ de MS consumida.

O consumo médio diário de água, expresso em litros/kg MS, observado neste trabalho, foi de 3,88 litros, dentro do intervalo recomendado por Frape (1998), de 2 a 4 litros/kg MS, e por Cymbaluk (1989), que observou consumo de 2,7 a 5,5 litros de água/100 $\mathrm{kg}$ de peso vivo para cavalos mantidos em baias sob temperaturas moderadas, entretanto foi superior ao observado por Pearson \& Merritt (1991), de 1,92 litros/kg MS. O valor de 3,42 litros de água ingeridos por eqüinos consumindo dieta com $100 \%$ de feno foi ligeiramente inferior ao observado por Fonnesbeck (1968), de 3,6 litros de água/kg MS, e ligeiramente superior ao observado por Cymbaluk (1989), de 3,2 L/kg MS, para animais consumindo dietas exclusivas de feno de gramíneas.

A excreção fecal de água foi maior nos animais ingerindo feno de gramínea do que nos animais alimentados com dietas mistas, de 1,74 e 0,83 litros de água/kg de MS ingerida, respectivamente. Por outro lado, Nyman \& Dahlborn (2000) observaram ingestão de água em eqüinos alimentados com dietas mistas de feno e aveia entre 43 e $58 \mathrm{~mL} / \mathrm{kg}$ de peso vivo.

Observou-se aumento linear $(\mathrm{P}<0,05)$ na excreção

R. Bras. Zootec., v.32, n.1, p.140-149, 2003 
Tabela 3 - Médias e equações de regressão (ER) dos valores diários de consumo médio de água, excreção fecal de água, excreção urinária de água, excreção total de água e balanço hídrico em eqüinos alimentados com diferentes níveis de volumoso na dieta

Table 3 - Averages and regression equations (RE) of daily water intake, fecal water, urinary water, total excretion of water and water balance in horses fed different levels of roughage in diets

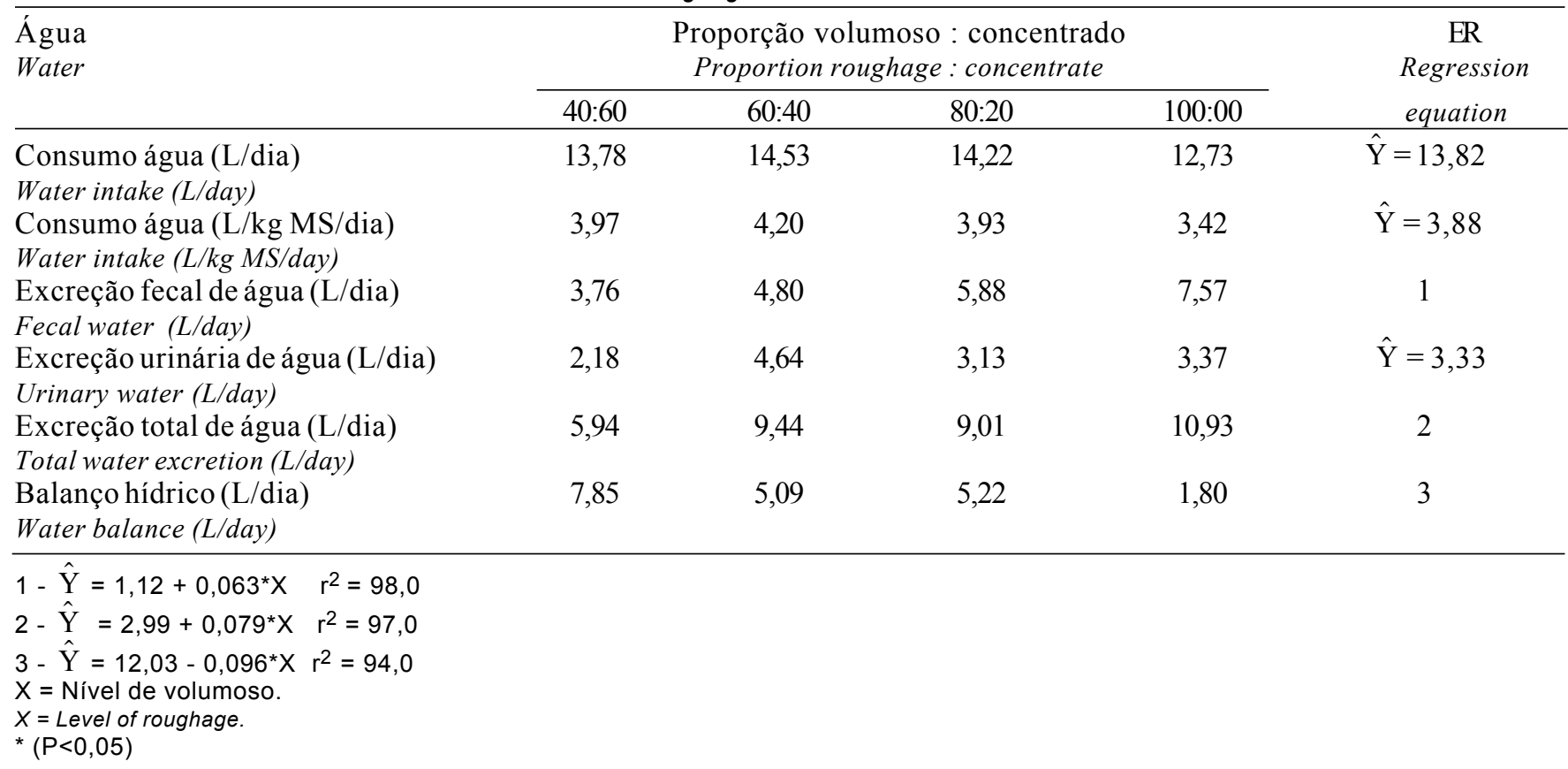

de água nas fezes, com o aumento do nível de volumoso na dieta, resultados semelhantes aos observados por Cymbaluk (1989), avaliando eqüinos alimentados com dietas constituídas exclusivamente por feno de gramínea ou leguminosa ou associados à ração concentrada. No entanto, a excreção de água através da urina não foi influenciada pelo nível de volumoso na dieta. A excreção total de água aumentou de forma linear $(\mathrm{P}<0,05)$ com a inclusão de volumoso na dieta, efeito que pode ser atribuído à excreção fecal de água.

Observou-se redução linear no balanço hídrico (BH), com o aumento do nível de volumoso na dieta, segundo a equação BH $\hat{Y}=12,03-0,096 * X, r^{2}=94,0$ $(\mathrm{P}<0,05)$, em que $\mathrm{X}$ é o nível de volumoso na dieta, indicando que animais alimentados com dietas contendo maior proporção de concentrado retêm mais água no organismo que aqueles alimentados com dietas contendo maior proporção de volumoso.

Os valores dos compostos nitrogenados ingeridos (NI), fecal ( $\mathrm{N}$ fecal), urinário (Nurina), absorvido (NA) e retido (NR) e suas equações de regressão ajustadas em função do nível de volumoso nas dietas são apresentados na Tabela 4.

Observou-se efeito quadrático sobre a quantidade diária de NI na dieta com o aumento do nível de volumoso, de acordo com a equação $\hat{\mathrm{Y}}=-11,934+$ $2,345 \mathrm{X}-0,01738 \mathrm{X} 2\left(\mathrm{R}^{2}=82,0\right)$, ocorrendo um aumento máximo estimado no nitrogênio ingerido quando o nível de volumoso na dieta for de $67,46 \%$.

Observou-se aumento linear positivo $(\mathrm{P}<0,05)$ na quantidade de $\mathrm{N}$ fecal, com o aumento no nível de volumoso na dieta, e, considerando-se a dieta exclusiva em volumoso, ocorreu menor consumo de $\mathrm{N}$ em relação às demais dietas. Tal fato pode estar relacionado com o aumento na excreção endógena de compostos nitrogenados, em função da elevação do conteúdo de fibra dietética. Em outros estudos sobre balanço de nitrogênio em eqüinos, observou-se aumento do $\mathrm{N}$ fecal, em função do aumento do $\mathrm{N}$ ingerido (Slade et al., 1970; Hintz \& Schryver, 1972; Prior et al., 1974).

Observou-se efeito quadrático do nível de volumoso na dieta sobre a excreção urinária de $\mathrm{N}$, de acordo com a equação $\hat{\mathrm{Y}}=-13,89+1,479 \mathrm{X}$ $0,01205 \mathrm{X} 2\left(\mathrm{R}^{2}=99,0\right)(\mathrm{P}<0,05)$, ocorrendo aumento máximo no $\mathrm{N}$ urinário, quando o nível de volumoso foi de $61,37 \%$ na dieta. Este comportamento esteve concordante com o $\mathrm{N}$ ingerido diário, que também apresentou comportamento quadrático, parecendo haver proporcionalidade entre a quantidade ingerida e a quantidade excretada de nitrogênio através da urina, 
Tabela 4 - Médias e equações de regressão (ER) dos compostos nitrogenados (N) ingerido (NI), N fecal (NF), N urinário (NU), $\mathrm{N}$ urina (\% $\mathrm{NI}$ ), $\mathrm{N}$ retido (NR), $\mathrm{N}$ absorvido (NA), $\mathrm{N}$ retido (\% $\mathrm{NI}$ ) e $\mathrm{N}$ retido (\% $\mathrm{NA}$ ), em função do nível de volumoso nas dietas

Table 4 - Averages and regression equations (RE) of intake nitrogen (NI), fecal $N(F N)$, urinary $N(U N)$, urinary $N(\% N I)$, retained $N(R N)$, absorbed $N(A N)$, retained $N(\% N I)$ and retained $N(\% A N)$ in function of roughage levels in diets

\begin{tabular}{|c|c|c|c|c|c|}
\hline \multirow[t]{2}{*}{$\begin{array}{l}\text { Nitrogênio } \\
\text { Nitrogen }\end{array}$} & \multicolumn{4}{|c|}{$\begin{array}{l}\text { Proporção volumoso: concentrado } \\
\text { Proportion roughage: concentrate }\end{array}$} & \multirow{2}{*}{$\begin{array}{c}\text { ER } \\
\text { Regression } \\
\text { equation }\end{array}$} \\
\hline & $40: 60$ & $60: 40$ & $80: 20$ & $100: 00$ & \\
\hline $\begin{array}{l}\mathrm{N} \text { ingerido }(\mathrm{g} / \mathrm{dia}) \\
\text { Intaked } N(\mathrm{~g} / \text { dav })\end{array}$ & 55,93 & 62,24 & 69,69 & 48,19 & 1 \\
\hline $\begin{array}{l}\mathrm{N} \text { fecal (g/dia) } \\
\text { Fecal N (g/day) }\end{array}$ & 20,31 & 18,69 & 24,42 & 27,07 & 2 \\
\hline $\begin{array}{l}\mathrm{N} \text { urina }(\mathrm{g} / \text { dia }) \\
\text { Urinary } N(g / \text { day })\end{array}$ & 25,63 & 32,54 & 26,24 & 13,87 & 3 \\
\hline $\begin{array}{l}\mathrm{N} \text { urina }(\% \mathrm{NI}) \\
\text { Urinary } N\end{array}$ & 44,54 & 54,59 & 36,84 & 31,24 & 4 \\
\hline $\begin{array}{l}\mathrm{N} \text { retido (g/dia) } \\
\text { Retained } N(\mathrm{~g} / \text { day })\end{array}$ & 10,00 & 11,01 & 19,04 & 7,24 & $\hat{\mathrm{Y}}=11,82$ \\
\hline $\begin{array}{l}\mathrm{N} \text { absorvido (g/dia) } \\
\text { Absorbed } N(\text { g/day })\end{array}$ & 35,63 & 43,54 & 45,27 & 21,11 & 5 \\
\hline $\begin{array}{l}\mathrm{N} \text { retido }(\% \mathrm{NI}) \\
\text { Retained } N\end{array}$ & 19,47 & 16,69 & 27,70 & 14,87 & $\hat{\mathrm{Y}}=19,68$ \\
\hline $\begin{array}{l}\mathrm{N} \text { retido (\% NA) } \\
\text { Retained } N\end{array}$ & 28,98 & 23,72 & 43,24 & 33,91 & $\hat{\mathrm{Y}}=32,46$ \\
\hline
\end{tabular}

$1-\hat{Y}=-11,934+2,345^{*} X-0,01738^{*} X^{2} ; R^{2}=82,0$

2- $\hat{Y}=13,51+0,13^{*} X ; r^{2}=77,0$

$3-\hat{Y}=-13,89+1,479^{*} X-0,01205^{*} X^{2} ; R^{2}=99,0$

$4-\hat{Y}=61,97-0,2881^{*} X ; r^{2}=54,0$

$5-\hat{Y}=-37,19+2,5976^{*} X-0,02004^{*} X^{2} ; R^{2}=95,0$

$\mathrm{X}=$ Nível de volumoso.

$X=$ Level of roughage.

* $(P<0,05)$.

havendo, assim, equilíbrio no balanço de nitrogênio.

Em outros estudos (Reitnour \& Treece, 1971; Prior et al., 1974; Almeida et al., 1998), avaliando-se níveis crescentes de inclusão de proteína nas dietas, observou-se maior excreção de compostos nitrogenados na urina, quando houve aumento do nível protéico nas dietas. Segundo Meyer et al. (1985), o metabólito de maior excreção de nitrogênio na urina é a uréia proveniente do metabolismo hepático da amônia absorvida no intestino grosso e, também, oriunda do catabolismo dos aminoácidos.

Freeman et al. (1988), avaliando o balanço de nitrogênio em cavalos em exercício, observaram aumento da excreção urinária de $\mathrm{N}$, quando a quantidade de $\mathrm{N}$ ingerido foi aumentada, semelhantemente ao observado no presente trabalho. $\mathrm{O}$ aumento nas perdas de N urinário também foi reportado por Slade et al. (1970), com cavalos em mantença alimentados com níveis dietéticos crescentes de nitrogênio.

Observou-se redução linear $(\mathrm{P}<0,05)$ no $\mathrm{N}$ urinário, expresso em percentual do $\mathrm{N}$ ingerido, com o aumento do nível de volumoso da dieta, segundo a equação $\hat{\mathrm{Y}}=61,97-0,2881 * \mathrm{X}(\mathrm{r} 2=54,0)$, em que o menor valor observado foi de $31,24 \%$ nos eqüinos consumindo dietas exclusivas em volumoso e o maior, de $54,59 \%$ nos eqüinos alimentados com dietas contendo $60 \%$ de volumoso. Entretanto, não houve efeito significativo dos diferentes níveis de volumoso nas dietas sobre a quantidade diária de $\mathrm{N}$ retido, observando-se valor médio de 11,82 g/dia.

Em relação à quantidade de $\mathrm{N}$ absorvido, observou-se efeito quadrático, em função do nível de volumoso na dieta, de acordo com a equação $\hat{\mathrm{Y}}=$ $37,19+2,5976 \mathrm{X}-0,02004 \mathrm{X} 2(\mathrm{R} 2=95,0)(\mathrm{P}<0,05)$. A estimativa da absorção máxima do $\mathrm{N}$ no trato gastrointestinal será obtida quando o nível de volumoso atingir a proporção de $64,8 \%$ na dieta.

Quanto à quantidade de $\mathrm{N}$ retido expresso em percentual do $\mathrm{N}$ ingerido ou do $\mathrm{N}$ absorvido, não foram observados efeitos do nível de volumoso na dieta $(\mathrm{P}>0,05)$, apresentando valores médios de 19,68 e $32,46 \%$, respectivamente. Prior et al. (1974) rela- 
taram valores de $\mathrm{N}$ retido de $28,3 \%$ do $\mathrm{N}$ absorvido, em eqüinos alimentados com dietas contendo $13 \%$ de $\mathrm{PB}$, resultado semelhante ao observado no presente trabalho para os animais consumindo dieta com $40 \%$ de volumoso.

\section{Conclusões}

O nível de volumoso nas dietas não afetou o tempo médio de retenção e a taxa de passagem da digesta, enquanto o tempo de trânsito da fase líquida da digesta reduziu de forma linear, em função do aumento do nível de volumoso na dieta. O tempo médio de retenção e o tempo de trânsito da digesta foram maiores para a fase sólida, em comparação à fase líquida da digesta dos eqüinos consumindo dietas com diferentes níveis de volumoso.

O nível de volumoso na dieta não influenciou o consumo de água, no entanto, a excreção de água nas fezes aumentou de forma linear, com a inclusão de volumoso na dieta, influenciando o balanço hídrico, que reduziu de forma linear, com a inclusão de volumoso na dieta.

No balanço de nitrogênio, as quantidades de compostos nitrogenados ingeridos, excretados na urina e absorvidos no trato digestivo foram afetadas em função do nível crescente de volumoso nas dietas, com valores máximos esperados, quando a inclusão de volumoso na dieta for de 67,$7 ; 61,4$; e $64,8 \%$, respectivamente.

\section{Literatura Citada}

ALMEIDA, F.Q. Fluxo de matéria seca ileal, perdas endógenas e digestibilidade pré-cecal e total da proteína e aminoácidos em eqüinos. Viçosa, MG: Universidade Federal de Viçosa, 1997. 120p. Tese (Doutorado em Zootecnia) - Universidade Federal de Viçosa, 1997.

ALMEIDA, F.Q.; VALADARES FILHO, S.C.; QUEIROZ, A.C. et al. Digestibilidade aparente e verdadeira pré-cecal e total da proteína em dietas com diferentes níveis protéicos em eqüinos. Revista Brasileira de Zootecnia, v.27, n.3, p.521-529, 1998.

BOLIN, D.W.; KING,R.P. A simplified method for determination of $\mathrm{Cr}_{2} \mathrm{O}_{3}$ when used as an indicator. Science, v.116, p.634, 1952 .

CUDDEFORD, D.; PEARSON, R.A.; ARCHIBALD, R.F. et al. Digestibility and gastro-intestinal transit time of diets containing different proportions of alfalfa and oat straw in Thoroughbreds, Shetland ponies, Highland ponies and donkeys. Animal Science, v.61, p.407-417, 1995.

CUDDEFORD, D.; WOODHEAD, A.; MUIRHEAD, R. A comparison between the nutritive value of short-cutting cycle, high temperature-dried alfalfa and tymothy hay for horses. Equine Veterinary Journal, v.24, n.2, p.84-89, 1992.
CUNHA, T.J. Horse feeding and nutrition. 2.ed. San Diego: Academic Press, 1991. 445p.

CYMBALUK, N.F. Water balance of horses fed various diets. Equine Practice, v.11, n.1, p.19-24, 1989.

DROGOUL, C.; TISSERAND, J.L.; PONCET, C. Feeding ground and pelleted hay rather than chopped hay to ponies. 2 Consequences of fibre degradation in the cecum and the colon. Animal Feed Science and Technology, v.87, p.131-145, 2000.

FAICHNEY, G.J. The use of markers to partition digestion within the gastrointestinal tract of ruminants. In: MAcDONALD, I.W.; WARNER, A.I.C. (Eds). Digestion and metabolism in ruminant. Armidale: University of New England Publishing Unit, 1975. p.277-291.

FOMBELLE, A.; JACOTOT, E.; DROGOUL, C. et al. Effect of the hay:grain ratio on digestive physiology and microbial ecosystem in ponies. In: EQUINE NUTRITION AND PHYSIOLOGY SYMPOSIUM, 16., 1999, North Caroline. Proceedings... North Carolina:ENPS, 1999. p.151.

FONNESBECK, P.V. Digestion of soluble and fibrous carbohydrate of forage by horses. Journal of Animal Science, v.27, n.5, p.1336-1344, 1968.

FRAPE, D.L. Equine nutrition and feeding. Harlow: Longman Scientific Technical, 1992.373p.

FREEMAN, D.W.; POTTER, G.D.; SCHELLING, G.T. et al. Nitrogen metabolism in mature horses at varying levels of work Journal of Animal Science, v.66, p.407-412, 1988.

GLADE, M.J.; BELL, P.I. Lower digestive tract fermentation rates and nitrogen utilization in horses. In: EQUINE NUTRITION AND PHYSIOLOGY SYMPOSIUM, 6., 1981, Texas. Proceedings... Texas: ENPS, 1981.

GLADE, M.J. The influence of dietary fiber digestibility on the nitrogen requirements of mature horses. Journal of Animal Science, v.58, n.3, p.638-646, 1984.

GOMES, B.V. Influência das características químicas e físicas das forragens sobre o consumo, degradação e cinética da digesta ruminal. Viçosa, MG: Universidade Federal de Viçosa, 1991. 115p. Tese (Doutorado em Zootecnia) - Universidade Federal de Viçosa, 1991.

HINTZ, H.F.; SCHRYVER, H.F. Nitrogen utilization in ponies Journal of Animal Science, v.34, n.4, p.592-595, 1972.

HINTZ, H.F.; SCHRYVER, H.F.; LOWE, J.E. Digestion in the horse. Feedstuffs, v.45, n.27, p.25-26, 1973.

HOLLAND, J. L.; KRONFELD, D.S.; SKLAN, D. et al. Calculation of fecal kinetics in horses fed hay or hay and concentrate. Journal of Animal Science, v.76, p.19371944, 1998.

KIMURA, F.T.; MILLER, V.L. Improved determination of chromic oxide in cow feed and feces. Agricultural Food Chemical, v.5, p.216-217, 1957.

MEYER, H. Alimentação de cavalos. São Paulo: Varela, 1995 303p.MEYER, H. Nutrition of the equine Athete. In: EQUINE EXERCICE PHYSIOLOGY, 2., 1987, Davis. Proceedings... Davis: 1987, p. 644-673.

EYER, H.; Von STEIN, S.; SCHMIDT, M. Investigations to determine endogenous faecal and renal losses in horses. In: EQUINE NUTRITION AND PHYSIOLOGY SIMPOSIUM, 8., 1985, East Lansing. Proceedings ... East Lansing: ENPS, 1985. p.68-72.

NATIONAL RESEARCH COUNCIL - NRC. Nutrientes requeriments of horses. 5.ed. Washington, D.C.: 1989. 100p.

NYMAN, S.; DAHLBORN, K. Effect of water supply method and flow rate on drinking behavior and fluid balance in horses. Physiologic Behavior, v.73, p.1-8, 2000.

R. Bras. Zootec., v.32, n.1, p.140-149, 2003 
OLSSON, N.; RUUDVERE, A. The nutrition of the horse. Nutrition Abstract and Reviews, v.25, n.1, p.1-18, 1955.

PAGAN, J.D. Forages for horses: more than just filler. In: PAGAN, J.D. (Ed.) Advances in equine nutrition II. Versailles, Kentucky: Kentucky Equine Research, 2001. p.13-28.

PAGAN, J.D.; HARRIS, P.A.; BREWSTER-BARNES, T. et al. Exercise affects digestibility and rate of passage of all-forage and mixed diets in thoroughbreds horses. Journal of Nutrition, v.128, p.2704S-2707S, 1998.

PEARSON, R.A.; MERRIT, J.B. Intake, digestion and gastrointestinal transit time in resting donkeys and ponies and exercised donkeys given ad libitum hay and straw diets temperature-dried alfafa and tymothy hay for horses. Equine Veterinary Journal, v.23, n.5, p.339-343, 1991.

PRIOR, R.L.; HINTZ, H.F.; LOWE, J.L. et al. Urea recycling and metabolism of ponies. Journal of Animal Science, v.38, n.3, p.565-571, 1974.

REITNOUR, C.M.; TREECE, J.M. Relationship of nitrogen source to certain blood components and nitrogen balance in the equine. Journal of Animal Science, v.32, n.3, p.487490, 1971.

SILVA, D.J. Análise de alimentos (métodos químicos e biológicos). 2.ed. Viçosa, MG: Universidade Federal de Viçosa, 1990. $166 \mathrm{p}$.

SLADE, L.M.; ROBINSON, D.W.; CASEY, K.E. Nitrogen metabolism in nonruminant herbivores. 1 - The protein influence of non protein nitrogen and protein quality on the nitrogen retention of adult mares. Journal of Animal Science, v.53, n.3, p.753-760, 1970.

TASKER, J.B. Fluid and electrolyte studies in the horse. II. An apparatus for the collection of total daily urine and faeces from horses. Cornell Veterinarian, v.56, n.1, p.77-84, 1966.

TODD, K.L.; SAUER, W.C.; CHRISTOPHERSON, R.J. et al. The effect of level feed of intake on nutrient and energy digestibilities and rate of feed passage in horses. Journal of Animal Physiology and Animal Nutrition, v.73, p.140-148, 1995.
UDÉN, P.; COLUCCI, P.E.; Van SOEST, P.J. Investigation of chromium, cerium and cobalt as markers in digesta rate of passage studies. Journal of Science and Food Agricultural, v.31, p.625-632, 1980.

UDÉN, P.; ROUNSAVILLE, T.R.; WIGGANS, G.R. et al. The measurement of liquid and solid ingesta retention in ruminants, equine and rabbits given timothy (Phleum pratense) hay. British Journal of Nutrition, v.48, n.2, p.329-339, 1982.

UNIVERSIDADE FEDERAL DE VIÇOSA - UFV. SAEG Sistema de análises estatísticas e genéticas (Manual do usuário). Viçosa, MG: Universidade Federal de Viçosa, 1997. 150p. (versão 7.0).

VANDER NOOT, G.W.; GILBRAITH, E.B. Comparative digestibility of components of forages by geldings and steers. Journal of Animal Science, v.31, p.351-355, 1970.

WANDERLEY, R.C.; MANZANO, A; ESTEVES, S.N. et al. Cinética da passagem da digesta em eqüinos. In: REUNIÃO ANUAL DA SOCIEDADE BRASILEIRA DE ZOOTECNIA, 29., 1992. Lavras. Anais... Lavras: Sociedade Brasileira de Zootecnia, 1992. p.284.

YONDER, D.E.; MILLER, E.; ROOK, J.E. et al. Fibre level and form: effects on digestibility, digesta flow and incidence on gastrointestinal disorders. In: EQUINE NUTRITION AND PHYSIOLOGY SIMPOSIUM, 15., 1997, Forth Worth. Proceedings... Texas: ENPS, 1997. p.122-127. 